Revista de Psicología de la PUCP. Vol. XVII, 2, 1999.

\title{
LA PSICOLOGÍA EN AMÉRICA LATINA EN EL SIGLO XX: UN ANÁLISIS DE SUS CARACTERÍSTICAS
}

\author{
Reynaldo Alarcón' \\ Universidad Ricardo Palma
}

Con el propósito de identificar las características más relevantes que tipifican a la psicología latinoamericana hacia fines del siglo XX, se señalan y discuten seis notas dominantes: su orientación científica, el carácter dependiente, la escasa originalidad, la relevancia social y su permeabilidad política, la preferencia por la investigación aplicada y su interés por el hombre como objeto central de sus trabajos. Se señala como limitación para establecer generalizaciones, el que las observaciones se basan solamente en las naciones latinoamericanas más activas en la generación de conocimientos psicológicos.

Palabras claves: psicología, latinoamérica, dependencia, originalidad, política, investigación aplicada.

The Latin American psychology in the XX century: An analysis of its characteristics

In order to identify the most important characteristics that describe the Latin American Psychology at the end of the century, six main issues are discussed: the scientific orientation, the dependency characteristic, the lack of an original approach, the social meaning, the politic permeability, the emphasis on applied research, and the interest in the person as the central object of study. The fact that these observations are based on the more active nations in the generation of psychological knowledge could be a limitation in the generalization of the findings.

Key words: psychology, latinamerican, dependency, originality, politic, applied research.

1. Psicólogo, doctor en filosofia y psicología. Es profesor emérito de la Universidad Nacional Mayor de San Marcos, profesor principal de la Universidad Ricardo Palma y de la Escuela de Postgrado de la Universidad Femenina. Ha publicado numerosos trabajos de investigación empírica y artículos reóricos sobre temas psicológicos. Es miembro del Comité Editorial de la Revista Latinoamericana de Psicología (Bogotá), de la Junta de Consultores Editoriales de la Revista Intercontinental de Psicología y Educación (México) y miembro del Consejo Asesor de Cuadernos Argentinos de Historia de la Psicologia. Correo electrónico: rcalarcon@computextos.net. 

El presente trabajo busca identificar las características de mayor relieve que acusa la psicología en América Latina, hacia fines del siglo $\mathrm{XX}$, en un intento de aislar lo que riene de común, más allá de las diferencias que puedan existir entre los países. El propósito es arribar a generalizaciones que la tipifiquen, que puedan dar cuenta de cómo es la psicología que se cultiva en la región. Las limitaciones de los hallazgos están dadas por los diferentes niveles de desarrollo de la psicología en los países latinoamericanos, al punto que las notas observadas puedan corresponder a unos pocos países, cuya presencia en la producción de conocimientos psicológicos es muy significativa.

El problema referente a la caracterización de la psicología latinoamericana no es nuevo, aunque no se han sistematizado sus notas. Han contribuido a seńalar sus características Ardila (1986a, 1992), Quiñones et al. (1991), Klappenbach y Pavesi (1994), de la Torre Molina (1995) y Alarcón (1997 a, 1997b). Estas contribuciones las comentaremos oportunamente. Utilizamos como fuentes para esta indagación los artículos de investigación publicados en revistas de psicología de la región, los resúmenes de las ponencias de los congresos interamericanos de psicología, evaluaciones sobre la psicología latinoamericana y libros de autores latinoamericanos. Hemos logrado identificar seis características esenciales de la psicología en América Latina, que seguidamente pasamos a comentarlas.

\section{La orientación científica}

Probablemente el interés dominante de los pioneros europeos de la psicología en América del Sur, fue hacer de la psicología una ciencia empírica, liberada de la Filosofía. Este interés se observa en Radecki, 
Blumenfeld, Mira y López, Mercedes Rodrigo y Helena Antipoff; y en los argentinos Horacio Piñero, Victor Mercante, José Ingenieros, Enrique Mouchet, Alfredo Calcagno; así como en los brasileños Lourenco Filho, Noemí Silveira de Rudolfer; y, en los mexicanos Ezequiel Chávez, Jesús Pacheco y Rafael Serrano. Los pioneros europeos afincados en América del Sur fundaron laboratorios de psicología experimental, divulgaron el mérodo científico, que utilizaron en sus trabajos, trabajaron con tests psicológicos y pedagógicos, hicieron uso de métodos estadísticos, impulsaron el movimiento psicométrico y los estudios de desarrollo y, varios de ellos escribieron, también, estudios filosóficos, aunque manteniendo la autonomía de la psicología.

La influencia de los pioneros, a través de sus publicaciones o desde sus cátedras de psicología, fue decisiva para establecer una psicología objetiva, empírica y experimental, separada de la Filosofía, como la década de los años 30, de fuerte predominio del intuicionismo de Bergson, de la fenomenología de Husserl y de otras expresiones del idealismo alemán. En algunos países, como el Perú, la oposición a la introducción de la psicología objetiva fue muy fuerte. En los círculos filosóficos, que dominaban en la enseñanza de la psicología, había un cierto temor de que volviera el positivismo, que había sido rechazado, hacía no más de una década. Sólo llega a aceptarse la psicología científica a mediados de los años 50 , cuando se establece la carrera de psicología.

En la actualidad, nadie discute el carácter científico de la psicología, más bien se ha preguntado, qué clase de ciencia es. ¿Es una ciencia natural, como fue el manifiesto deseo de los iniciadores del movimiento separatista de la Filosofía? $\mathrm{O}$ ¡es una ciencia social?, propuesta mucho más reciente. Lo cierto es que la amplitud de áreas de la psicología actual, la pueden ubicar en una u otra rama de las ciencias. La psicofisiología puede ubicársele en el grupo de las ciencias biológicas, y la psicología social se acerca más a las ciencias sociales.

Hablar de una psicología independiente de la filosofía no significa rechazar a ésta; lo que ha significado para los psicólogos ha sido, en 134 
primer término, sacar su objeto del estudio de la temática filosófica, interesada en describir y captar la esencia de los fenómenos de la mente; y en segundo término, aplicar el método científico para estudiar los procesos psíquicos y el comportamiento. Es innegable la relación entre psicología y filosofía. Todas las orientaciones de la psicología contemporánea, sea el conductismo skinneriano, la psicología cognitiva y la psicología humanística se apoyan en sistemas filosóficos; asimismo, el método científico utilizado por la psicología, tiene sus raíces en el antiguo empirismo de Bacon.

La investigación psicológica latinoamericana ha adoptado el método científico. Es predominantemente empírica, objetiva y cuantitativa, hace uso de instrumentos objetivos para recoger sus datos, con un claro predominio de tests, escalas, inventarios y cuestionarios. Asimismo, hace poco uso de instrumental de laboratorio (Alarcón, 1997b). El tratamiento de los datos se hace mediante pruebas estadísticas, cada vez más sofisticadas, aunque muy poco se utilizan modelos multiecuacionales causales. En los trabajos predomina el paradigma hipotético-deductivo, orientado a la verificación de hipótesis; el conductismo operante rechazó este paradigma y reactualizó la tradición empírica inductivista de Bacon y Hume, amparándose en las formulaciones del empirismo lógico del Círculo de Viena. Si bien este es el carácter dominante de la investigación psicológica que se realiza actualmente en América Latina, hay núcleos muy fuertes de psicólogos de orientación psicoanalítica, particularmente en Argentina, donde esta orientación es dominante. Por otro lado, en recientes décadas se advierten entusiasmos por la Psicología Humanística, la cual no rechaza de plano el método científico, pero no le otorga la misma importancia que tiene en las psicologías objetivas. Su insistencia en estudiar la vivencia directa e inmediata como base de los conocimientos, la ha llevado a que prefiera el enfoque fenomenológico para sus investigaciones. De estos dos últimos enfoques son muy escasos los trabajos que se publican en las revistas de psicología más conocidas de la región. 


\section{El carácter dependiente}

En un trabajo nuestro (Alarcón, 1997a), señalamos que América Latina había sido muy receptiva y magnífica anfitriona de doctrinas importadas, comportándose como subsidiaria del pensamiento europeo y después del anglo-americano. Esta situación no es reciente. Nuestras universidades coloniales impartieron conocimientos filosófico-psicológicos desde un enfoque escolástico, orientación ésta que dominó más de una centuria. Cuando soplaron vientos de renovación intelectual, se difundieron el racionalismo cartesiano, el empirismo de Locke y el sensualismo de Condillac. En los primeros años republicanos fueron el eclecticismo de Cousin, la ideología de Destutt de Tracy, la filosofía del sentido común de Thomas Reid, el idealismo de Krause; y a mediados del siglo pasado, el positivismo de Comte logra captar las inteligencias más lúcidas de nuestros países, abrazándolo como un credo, al punto que orientó el destino político de algunos países latinoamericanos. Cuando el positivismo es cuestionado y rechazado, con mucha vehemencia, por su posición antimetafísica, Bergson y su vitalismo es saludado como el salvador del pensar filosófico auténtico. En ninguna parte del mundo se acogió a Bergson con más devoción y entusiasmo que en América Latina, señala Barboza (1939). La filosofía de Bergson no fue recibida como un sistema, sino como un mensaje. Así lo recibieron Caso en México, Korn en Argentina y Deustua en el Perú. El dogmatismo cientificista del positivismo fue reemplazado por el dogmatismo espiritualista de Bergson al que se sumó después el pensamiento idealista germano. A estas doctrinas filosóficas, desde cuyos parámetros se enseñó psicología, le siguió, pasada la Segunda Guerra Mundial, la psicología norteamericana.

Todas estas tendencias recibieron adhesiones alborozadas, se les difundió sin espíritu crítico, se utilizaron sus teorías y postulados y más adelante sus tecnologías, sin someterlas a crítica ni verificación previa, poniéndose de lado las reglas del mérodo científico. Esto ha conducido a que en América Latina se haya desarrollado una psicología de claro matiz dependiente; una psicología refleja, condicionada por psicologías 136 
La psicologia en América Latina en el siglo XX: un análisis de sus características

extranjeras. Esta condición desdorosa fue denunciada por Rogelio DíazGuerrero (1971). En su discurso presidencial leído en el XII Congreso Interamericano de Psicología, celebrado en Montevideo, sostuvo:

"Creo que ha llegado el momento de que Iberoamérica se despoje de las cadenas del colonialismo de psicologías provenientes de Europa y Angloamérica. Ha llegado el momento de que, por un lado, sometamos a comprobación las afirmaciones que al respecto de la psicología humana en general se han hecho por autores alejados de la idiosincracia iberoamericana, $y$ ha llegado el momento de que desarrollemos nuestras propias concepciones al respecto de la estructura, del funcionamiento y de las características específicas de las personalidades de nuestros pueblos (Díaz-Guerrero, 1971, p. 6)".

El discurso de Díaz-Guerrero, meditado y vibrante, propone, para salir de la dependencia, el cuestionamiento racional de teorías y construcciones teóricas extranjeras, la contrastación científica de ellas y el desarrollo de ideas psicológicas propias, que corresponda a la idiosincracia de la gente de nuestros pueblos. Su actitud es eminentemente científica, libre de síntomas chauvinistas, tan en boga por los años en que efectuó su propuesta. Algunos psicólogos sociales (Marín, 1980, Montero y Blanco, 1992) han señalado que la investigación psicosocial en Latinoamérica, hasta fines de la década del 60 , se limitó a replicar los temas clásicos de la psicología internacional, advirtiéndose en ella un claro sentido dependiente, reproductora de los temas en boga en Norteamérica y Europa. En el curso de los años 70 se cuestiona esta situación y se pone en debate la significación social de sus hallazgos, planteándose hacer una disciplina de relevancia social, concepto que más adelante discuriremos, pero que apunta a efecruar investigaciones sobre problemas inmersos en la realidad de nuestros países, y que sus hallazgos puedan utilizarse a favor de los grupos latinoamericanos menos favorecidos.

La coyuntura política de los años 60 y 70 , alimentada por corrientes político-sociales de izquierda, dio pie a enjuiciamientos y rechazos de la 
psicología norteamericana. El punto de vista marxista ha sido expresado en años recientes por de la Torre Molina (1995), para quien la psicología norteamericana ha desempeñado un doble papel: por un lado promovió la exportación de modelos teóricos y su aplicación práctica; y por otro lado, contribuyó al sostenimiento del sistema social capitalista. Pero, el colonialismo cultural, vía la psicología norteamericana, expresamente el conductismo, que rechaza con vehemencia, es reemplazado con su adhesión a la psicología soviética, que representaba la psicología marxista. Esto significa, pasar de la dependencia cultural de un país y de un credo político a la dependencia de otro país y de otra psicología.

Más correcto nos parece rechazar la siruación de dependencia venga de donde venga, de cualquier nación poderosa, de derecha o de izquierda. Postulamos el desarrollo de una psicología latinoamericana crítica, abierta a los avances contemporáneos de la disciplina, más allá de las preferencias políticas de sus autores. La psicología latinoamericana superará la dependencia cultural en tanto se convierta en una activa generadora de conocimientos teóricos y tecnológicos, y esto sólo se puede conseguir mediante la investigación científica. Estimo que estamos en ese camino.

\section{Escasa originalidad}

Asociada al carácter dependiente o consecuencia de la misma, es la escasa originalidad que ha mostrado la psicología latinoamericana. Esta nota la advertimos cuando dimos cuenta del movimiento psicométrico en nuestros países, el cual orientó su trabajo a la adaptación de tests extranjeros y menos a la producción de pruebas originales (Alarcón, 1997 a). Atribuimos este hecho a la ausencia de teorías originales, sobre áreas del comportamiento a medir, desarrolladas en América Latina; como es sabido, los tests reposan en formulaciones teóricas basadas en la investigación básica. Los tests originales producidos en la región son el Test Miokinético de Mira y López, el Test ABC de Lourenco Filho, el Test rápido de Barranquilla, desarrollado por Francisco del Olmo, inspirado en el Test de Inteligencia de Otis, el Inventario de Intereses Vocacionales 
La psicología en América latina en el sigho XX: un análisis de sus características

de Arrigo Angelini, basado en el Inventario de Intereses de Thurstone, y el Test A-51 que construyó Walter Blumenfeld.

Actualmente, el trabajo psicométrico ha variado sustancialmente en varios países latinoamericanos. La idea de revisar las construcciones teóricas de los tests extranjeros fue bien acogida, particularmente en México, observándose en las últimas décadas un persistente interés por someter a rigurosas verificaciones estadísticas la validez de construcción de las pruebas. Esto ha conducido a rechazar más de una construcción teórica y reemplazarla con nuevas formulaciones de acuerdo a las características de los individuos de la cultura de adopción de un test; así como, también, a la producción de instrumentos originales.

Signos de que la ausencia de originalidad está en vías de quedar atrás, es la formulación de la teoría histórico-bio-psico-socio-cultural del comportamiento humano, de Rogelio Díaz-Guerrero (1972 a) y su Etnopsicologia, que denomina scientia nova, la cual busca desarrollar psicologías autóctonas, basada en los rasgos dominantes de los individuos de una sociocultura (Díaz-Guerrero, 1994). A estos dos trabajos se suma el libro de Rubén Ardila, Sintesis experimental del comportamiento (1988), en el que propone un paradigma unificador de la psicología. Más allá del acuerdo o desacuerdo con la propuesta, que tiene como base el conductismo operante, el paradigma de Ardila parte de premisas muy bien elaboradas, of reciendo un marco de referencia definido y sólido.

\section{Entre la relevancia social y la permeabilidad política}

Los psicólogos latinoamericanos se han mostrado muy sensibles a los problemas apremiantes de sus comunidades, donde la investigación o la intervención psicológicas puedan contribuir a su conocimiento o a su solución. El tema se puso en debate en el XI Congreso Interamericano de Psicología, que se reunió en México en 1967, que tuvo por título La contribución de las ciencias psicológicas y del comportamiento al desarrollo social y económico de los pueblos. Se buscaba conectar la indagación psicológica con los problemas de las sociedades subdesarrolladas, o, para 
expresarlo en otra forma, se trataba de orientar la investigación psicológica hacia problemas ligados al desarrollo social, se ponía énfasis en investigar problemas cuyos hallazgos pudieran ser útiles para facilitar el desarrollo del país, para comprender la conducta de los individuos que viven en ambientes deprivados y para favorecer intervenciones psicológicas relevantes para el desarrollo y bienestar humanos. Este interés ha sido motivado por la presencia de problemas de salud, desnutrición, analfabetismo, violencia política, terrorismo, trabajo infantil, delincuencia, drogadicción, frustración política y muchos otros problemas que acusan nuestros países.

En líneas anteriores señalamos que los psicólogos sociales plantearon hacer de la psicología una ciencia socialmente relevante, de suerte que sea una ciencia comprometida con los problemas propios de la realidad latinoamericana. También se interesaron en lograr estrategias que contribuyeran al desarrollo de las comunidades. Fruto de este interés fue el impulso que recibió la psicología social comunitaria, orientada hacia los sectores sociales menos favorecidos. Ésta acercaba al psicólogo y a la universidad donde trabajaba, a las comunidades marginales en un trabajo novísimo de investigación-acción.

La idea de relevancia social fue un poco más allá de su sentido originario, habiéndose postulado que la psicología, y más propiamente el psicólogo, debería asumir el compromiso político de combarir la estructura social vigente, calificada de injusta y opresora. Se alentó poner los conocimientos de la psicología al servicio de la liberación y del cambio político-social, buscando hacer de la psicología una ciencia políticamente compromerida. Ardila (1986a) observa que la psicología latinoamericana ha sido permeable a la utilización de categorías ideológicas y políticas; en tanto que Klappenbach y Pavesi, advierten que «la función social de la psicología, y la mejor manera de que el ejercicio de la profesión contribuyera a erradicar la injusticia, han sido cuestiones de permanente debate entre un número importante de aquellos que se dedican a la práctica de la psicología en América Latina» (Klappenbach y Pavesi, 1994, p. 468). Esta tendencia, según los mismos autores, "ha encontrado 
una vía de desarrollo profesional y académico, a través de la psicología política, la cual, en la región, ha combinado el análisis científico con el compromiso de transformación social». No está demás recordar que son muchos psicólogos los que han sufrido persecución política, destierro, encarcelamiento y hasta la muerte, por su compromiso político La muerte trágica de Ignacio Martín Baró es un ejemplo de esta nota de la psicología latinoamericana.

\section{La preferencia por la psicología aplicada}

Cuando se analizan las ponencias presentadas en los congresos interamericanos de psicología, es fácil advertir la orientación aplicada de la investigación, sea por los problemas que se abordan o por los métodos de investigación que se utilizan con mayor frecuencia. En los congresos de la SIP, celebrados en Santiago, en 1995 y en San Juan, en 1997 , predominaron los trabajos correlacionales $(37.9 \%)$ y exploratorios $(30 \%)$, las investigaciones experimentales apenas alcanzaron el 5\%. Los instrumentos para recoger datos fueron predominantemente tests objetivos, inventarios, escalas y cuestionarios. Los dispositivos experimentales fueron utilizados en sólo el $7 \%$ de los trabajos presentados. La investigación experimental que alcanzó un relativo auge en la década de los 70 y parte de los 80 , bajo la influencia del conductivo, ha sufrido un fuerte descenso.

En vista de estos datos, podría afirmarse que la investigación que se desarrolla en Latinoamérica, hacia fines del siglo XX, está más interesada en describir hechos y buscar relaciones de asociación entre variables medidas, que buscar relaciones causales entre variables manipuladas experimentalmente. Esta orientación no es nueva en la región, se haya presente en los trabajos de los pioneros europeos. No obstante que introdujeron laboratorios de psicología experimental, sus trabajos más representativos corresponden a la línea psicométrica y a estudios de desarrollo; los laboratorios fueron dedicados mayormente a la enseñanza de la psicología experimental. Esto no quiere decir que no se hizo 
investigación experimental, se hizo, pero en muy reducida proporción. Sin embargo, debe mencionarse que en la etapa pionera de la psicología sudamericana, se desarrolló en Argentina un entusiasta movimiento experimental promovido por Horacio Piñero, entre 1905 y 1914. Señala Papini (1976) que sobre un total de 56 trabajos realizados, los temas abordados fueron atención, memoria, psicofisiología, percepción y psicofísica.

En los comienzos de la etapa profesional de la psicología, la orientación aplicada continuó dominando y en la actualidad se mantiene. En busca de las causas probables, se puede argüir que los primeros trabajos psicométricos se debieron a la necesidad de contar con instrumentos válidos y confrables, para fines pedagógicos, que fue el espacio de aplicación inicial de la psicología. La psicometría latinoamericana actual tiene intereses más científicos. Su finalidad es revisar las bases teóricas en que reposan los tests, a fin de determinar si aquellas construcciones tienen validez en una sociocultura distinta a la de origen. También podría deberse al escaso entrenamiento en métodos de investigación avanzados que ofrecen nuestras universidades, o, debido a la falta de laboratorios experimentales, bien equipados, para la realización de investigaciones básicas.

La preferencia por la investigación aplicada en América Latina, probablemente obedezca al recurrente discurso de hacer una psicología conectada con los problemas reales de nuestras sociedades. Un documento de UNESCO publicado en 1979, señaló que el objetivo social de la ciencia y la tecnología es servir al desarrollo nacional y aumentar el bienestar de la humanidad en su conjunto. Pero además consideró que: "sería un desperdicio irracional de recursos que los países en desarrollo intenten generar conocimientos científicos y tecnológicos, sin aprovechar plenamente los conocimientos que ya se disponen gracias a la labor de científicos y tecnólogos de todo el mundo". El documento sugiere que los países del Tercer Mundo no hagan investigación básica y que más bien sigan utilizando los conocimientos producidos en los centros productores de conocimientos y tecnología. 
La antinomia entre investigación básica e investigación aplicada no es fácil de superar. La primera está íntimamente relacionada con el avance general de la ciencia, mientras que los conocimientos de la segunda, pueden dar pie a intervenciones psicológicas inmediatas, que pueden favorecer el desarrollo de los países y solucionar los problemas de los individuos de las comunidades empobrecidas. Los problemas de la invesrigación básica corresponden más a intereses científicos, aunque es difícil hablar de una ciencia aséptica, como antaño se supuso. De cualquier manera, la ciencia aplicada reposa en la ciencia básica. De allí nace el interés por realizar investigaciones básicas, aunque se ha dicho con alguna razón, que hacer investigación básica en el Tercer Mundo es hacer investigación por el prestigio (Alarcón, 1986).

En realidad, en el trasfondo de esta antinomia subyace una opción axiológica: se enfrentan valores puramente científicos a valores científicosociales. Por una u otra preferencia, la psicología larinoamericana se ha inclinado por el valor social de la ciencia.

\section{El hombre como problema central}

Una última nota dominante que hemos advertido es que la psicología latinoamericana tiene al hombre como problema central de sus investigaciones. Esto se diferencia de la psicología norteamericana que utiliza con frecuencia especies sub-humanas y extrapola sus hallazgos para explicar sectores de la conducta humana. Este es el caso del conductismo en el área del aprendizaje. Contrariamente, el psicólogo latinoamericano efectúa sus trabajos con sujetos humanos. Al colocar al hombre en el centro de su interés, coincide con la Psicología Humanística que hace lo propio, e insiste en que el ser humano que investiga sea parte de la investigación sobre el ser humano (Quitmann, 1989). Pero en la Psicología Humanística el método científico no tiene la misma relevancia que tiene en las psicologías objetivas, pues el método está subordinado al criterio de la experiencia humana. $\mathrm{Y}$ «en orden a estudiar la vivencia y el obrar humanos, prefiere un enfoque fenomenológico debido a la 
función relevante de la autoconsciencia como rasgo humano específicom (Auer, 1997, p. 50).

La psicología latinoamericana se ha interesado en el hombre, en describirlo, comprenderlo y explicarlo. Pero, más adelante, se ha interesado en conocer al hombre de estas tierras. Un ejemplo de este interés son los libros de Díaz-Guerrero (1972b) sobre psicología del hombre mexicano y el de Ruben Ardila (1986b) sobre el hombre colombiano. Este interés ha llevado a Díaz-Guerrero a fundar una corriente de investigación que denomina Etnopsicología, que tiene como programa desarrollar psicologías autóctonas. En este sentido, enfatiza lo idiosincrático, busca conocer los rasgos dominantes de la personalidad de los individuos de una sociocultura, de un pueblo, de una nación.

Afirmar que la psicología latinoamericana tiene al hombre como sujeto de sus investigaciones, no significa que no haya investigación comparada; lo que se quiere decir es que su producción es ciertamente exiguia. Tenemos la impresión de que el psicólogo latinoamericano tiene reparos para explicar la conducta humana a través de hallazgos obtenidos en especies sub-humanas; si lo que se busca es conocer al hombre en su medio y su circunstancia, en lo que tiene de básico y característico, por qué recurrir a explorar especies distintas a él. América Latina está poblada por una variedad étnica, donde habitan todas las razas del planeta. Es un laboratorio natural de razas y mestizajes aún por conocer; conocemos al hombre a través de la investigación de Europa y Norteamérica, pero dentro de la universalidad del hombre hay diferencias netas que impone el medio natural y la sociocultura. Conozcamos a los hombres de América Latina porque, con seguridad, todavía no los conocemos.

\section{Referencias}

Alarcón, R. (1986). Psicologia, pobreza y subdesarrollo. Lima: Editorial Educativa Inide.

Alarcón, R. (1997a). Orientaciones teóricas de la psicologia en América Latina. Lima: UNIFE. 
La psicología en América Latina en el siglo XX: un análisis de sus características

Alarcón, R. (1997b). La investigación psicológica en América Latina: un enfoque histórico. Conferencias/Conferences (pp. 16-31). Sao Paulo: Sociedad Interamericana de Psicología.

Ardila, R. (1986a). La psicología en América Latina: pasado, presente y futuro. México: Siglo XXI.

Ardila, R. (1986b). Psicología del hombre colombiano. Bogotá: Planeta.

Ardila, R. (1988). Sintesis experimental del comportamiento. Madrid: Alhambra.

Ardila, R. (1992). Entre el Río Grande y la Antártida. América Latina y su psicología a las puertas del siglo XXI. Libro de Ponencias (pp. 16-31). Madrid: Congreso Iberoamericano de Psicología.

Auer, H. (1997). Psicologia humanistica. Lima: UNIFE.

Barboza, E. (1939). Las ideas pedagógicas de Alejandro O. Deustua. Letras, 13, 161-178.

De la Torre Molina, C. (1995). Psicología latinoamericana. San Juan: Publicaciones Puertorriqueñas Editores.

Díaz-Guerrero, R. (1971). La enseñanza de la investigación en psicología en Iberoamérica: un paradigma. Revista Latinoamericana de Psicologia, 3, 5-36.

Díaz-Guerrero, R. (1972a). Hacia una teoría histórico.bio-psico.sociocultural del comportamiento humano. México: Trillas.

Díaz-Guerrero, R. (1972b). Estudios sobre psicología del mexicano. México: Trillas.

Díaz-Guerrero, R. y Pacheco, M. A. (Eds.) (1994). Etnopsicologia: scientia nova. República Dominicana: Corpio.

Klappenbach, H. A. y Pavesi, P. (1994). Una historia de la psicologia latinoamericana. Revista Latinoamericana de Psicologia, 26, 445482.

Marín, G. (1980). Presentación. Psicología Social (número monográfico). Revista Latinoamericana de Psicología, 12, 5-6.

Montero, M. y Blanco, A. (1992). La psicología social en el ámbito iberoamericano. Libro de Ponencias (pp.162-173). Madrid: Congreso Iberoamericano de Psicología. 
Papini, M. R. (1976). Datos para una historia de la psicología experimental argentina (hasta 1930). Revista Latinoamericana de Psicologia, 8, 319-335.

Quiñones Vidal, E., Vera Ferrándiz, J. A., Sánchez Hernández, A. y Predraja Linares, M. J. (1991). La segunda década de la Revista Latinoamericana de Psicología (1979-1988): una aproximación objetiva a la psicología en Latinoamérica. Revista Latinoamericana de Psicologia, 23, 155-182.

Quitmann, H. (1989). Psicologia humanistica. Barcelona: Herder. 